\title{
Crowdsourcing in Crisis and Disaster Management - Challenges and Considerations
}

\author{
Gerald Schimak, Denis Havlik, and Jasmin Pielorz \\ AIT Austrian Institute of Technology GmbH, Donau-City-Straße 1, 1220 Wien \\ \{Gerald.Schimak, Denis.Havlik, Jasmin.Pielorz\} @ait.ac.at
}

\begin{abstract}
With the rise of social media platforms, crowdsourcing became a powerful tool for mobilizing the public. Events such as the earthquake in Haiti or the downfall of governments in Libya and Egypt indicate its potential in crisis situations. In the scope of this paper, we discuss the relevance of crowdsourcing in the area of crisis and disaster management (CDM). Starting with a general overview of the topic, we distinguish between different types of crowds and crowdsourcing and define what is meant by crowdtasking in the area of CDM. After considering technological, societal and ethical challenges for using crowdsourcing in crisis management, applications of crowdsourcing tools in ongoing projects are described and future developments outlined.
\end{abstract}

Keywords: crowdsourcing, crowdtasking, data analysis, big data, social media, crises and disaster management, volunteers, volunteered geographic information.

\section{Introduction}

Crowdsourcing is rapidly gaining recognition as important source of information in crisis situations. The potential of crowdsourcing in crisis management is undeniably enormous: past experiences, e.g. in Haiti and Libya [1], show that information obtained through crowdsourcing is often more detailed and just as accurate as the information gathered through hardware sensors and through official channels. Successfully managed crowdsourcing can result in nearly instantaneous situation awareness (crisis mapping). However, crowdsourcing requires adequate tools, capable moderators and professional crisis managers that are willing and able to accept the crowdsourcing as an additional tool with its own advantages and limitations. A very good overview of the challenges related to crowdsourcing in crisis situations has been given in [2] and a very critical analysis of the four use cases which are often mentioned as best practices is given in [3].

After accepting crowdsourcing as a powerful crisis management tool, the next logical step for crisis managers is to attempt tasking the individual crowd members and smaller groups with well-defined activities. Properly organized crowdtasking can bring multiple benefits over spontaneous crowd self-organization. For the start, the available volunteer resources can be mobilized more rapidly and deployed more efficiently to provide information where it is missing or to quickly confirm and improve unreliable or imprecise reports. Information received through crowdtasking is therefore inherently 
much more reliable and also easier to interpret than information that has been gathered by data mining from existing social networks. In addition, citizens could be individually instructed to help themselves and their neighbors, taking into account real needs, as well as individual aptitudes and capabilities.

However, it is important to understand that the "crowd" is inherently nonhierarchical, has means to self-organize and will do so with or without crisis managers. This is a challenge for the crisis managers, since their professional experience indicates that crisis management requires a hierarchic organisation and that selforganization of inexperienced civilians can be outright dangerous in crisis situations. On the other hand, the attempt to overmanage the crowd could severely limit the number of ad-hoc volunteers willing to participate in the crowdtasked activities.

On the subject of "using the crowd as a valuable crisis management resource" we therefore distinguish between: (1) information and activities of individuals and selforganized groups, which are supported by general-purpose platforms such as Facebook or Waze $^{1}$ and (2) information and activities of loosely organized volunteer groups, whose activities are, to a certain extent, coordinated by professional crisis managers and supported by a dedicated mobile crowdtasking platform.

In section 2, we present some general remarks about crowdsourcing, thereafter we present several examples of crowdsourcing and crowdtasking at work, discuss key characteristics and challenges of crowdsourcing in crisis management and finally outline the generic methodology and functional architecture for future dedicated crisis management crowdtasking tools.

\section{Crowdsourcing in Crisis Management}

\subsection{What Is Crowdsourcing?}

According to [4], the term crowdsourcing was first coined by Jeff Howe, who used it in an article published in June 2006 for the Wired magazine. Following Howe's original conception of the term, we define crowdsourcing as a "method for recruiting and organizing ad-hoc labor, using an open-call for participation" [5]. Crucial to this definition is the notion of an extremely fluid workforce, one that is devoid of managerial and contractual directives inherent to other, more traditional labor models, such as "outsourcing." The crowd has the freedom to do what it pleases, when it pleases, and it is often up to designers to find clever ways to recruit and retain this kind of ad-hoc workforce.

Unlike the crowd itself, the entity requesting crowdsourced work is rarely fluid or loosely defined. Indeed, the request for work usually comes from a distinct organization or individual (known as the "requester", in crowdsourcing parlance). This distinguishes crowdsourcing from other, more decentralized labor structures, such as commons-based peer production, as well as from the "smart mobs" which use the social networking for self-organization [6]. While crowdsourcing can take many forms, three dominant types

https : / /www.waze.com/events 
of platforms have emerged in the past decade: (1) games with a purpose, (2) micro-task markets and (3) open innovation contests.

\subsection{Social Data Mining and Crowdsourcing in Crisis Management}

In the domain of crisis management, the term crowdsourcing usually refers to the gathering of information from the crowd in all phases of a crisis and results in socalled crowdsourced crisis mapping. The request for work in this type of crowdtasking usually remains at the level of general calls for action. In fact, the border towards social data mining is fuzzy because in this case data from general purpose social media (e.g. geo-tweets) is often used instead, or in addition to, the data received through dedicated platform such as Ushahidi ${ }^{2}$.

From an organizational point of view, social data mining is the simplest form of crowdsourcing. Information relevant to a crisis will automatically appear in the social media, even if nobody explicitly asks for it. However, the general purpose social networking platforms such as Facebook or Google+ lack some features which are important for the crisis managers.

To begin with, much of the information is shared only with friends and family and therefore cannot be used by crisis managers without raising privacy issues. If, however, people share their information with the public, automatic filtering and interpreting the available information (e.g. from twitter or from a fictive "friends of Red Cross" Facebook group) is challenging. When reporting about a crisis event, people can use any language, slang and choose a hashtag or topic they find relevant, making it very difficult to understand the actual content of a post. In addition, most posts or tweets currently have no geographical location tagged to them, so that a crisis manager cannot be sure about the exact location of an incident.

In order to circumvent these difficulties, crisis managers can organize an ad-hoc platform for gathering of information from the population. This has been successfully done in the past [1], and crisis mapper ${ }^{3}$ volunteers excel in using such information for ad-hoc mapping of the crisis development.

\subsection{Crowdtasking and Micro-task Markets}

In order to distinguish between this already established practice and more fine-grained crowdsourcing of both, information and more physical tasks, (e.g. "fill sacks with sand") in crisis situations, we use the word "crowdtasking" for the process, and "adhoc volunteers" for the participants.

Crowdtasking can be seen as a special form of a micro-task market. Both forms of crowdsourcing have in common that (1) organizers can request concrete and well-defined actions within a limited temporal and spatial scope; (2) participants are explicitly asked to

\footnotetext{
http: / /www.ushahidi.com/

http://crisismappers.net/
} 
perform specific micro-tasks; and (3) participants are not obliged, or even expected, to work beyond the concrete tasks they choose to complete.

However, the crowdtasking puts much stronger emphasis on space and time. Adhoc volunteers are asked to visit particular location(s), report requested state parameters (e.g. "is this house damaged?") using a mobile device and possibly perform some limited physical actions (e.g. "help the inhabitants to prepare for evacuation"). Due to the inherent dynamics of the crisis, the tasks are often urgent and the probability of endangering the ad-hoc volunteers is not negligible.

In order to optimize the tasking process and minimize the risk for volunteers, tasking organizations need to know the volunteers' capabilities and whereabouts. Therefore the volunteers need to enroll prior to action. In the case of Team Austria, volunteers can register themselves online at any time and indicate their skills and address. However, a verification of the data by Red Cross personnel is required to reach the status of a trusted volunteer. In this way, triggering and tasking can be done very efficiently and with a minimal risk for volunteers.

\section{Use Cases, Existing Systems or Applications}

In this chapter we present several examples of crowdsourcing and crowdtasking for crisis management. The examples are ordered by complexity of the tasking process considered, starting with a passive approach of gathering relevant information from social media platforms and ending with a prototypic implementation of a dedicated crowdtasking methodology and platform, which has been developed specifically for crisis management.

\subsection{Social Sensors for Security Assessments and Proactive Emergency Management}

Social media statistics such as 20 million tweets related to hurricane "Sandy" or disaster-related photos shared at a rate of $10 / \mathrm{sec}$ on Instagram provide tangible evidence that security forces and civil protection agencies could greatly benefit from the effective blending of social media information into their processes. Due to its versatility, social media could serve in the crisis management domain as both a valuable data source for first responders and an effective communication tool to reach the public. However, despite the widespread use of social media in various domains, e.g. in marketing or finance, there is still no structured and effective way to leverage several social media in crisis management applications.

In order to tackle this challenge, the EU FP7 project SUPER (Social sensors for security assessments and proactive emergencies management) ${ }^{4}$ aims at developing a holistic and privacy-friendly approach to exploit social media for emergencies and security incidents. In a joint effort of experts from the security and social media domain, the project addresses all phases of crisis management, i.e. before, during and

4 http://super-fp7.eu/ 
after a crisis occurred and will integrate social media within existing security and management systems for tactical operations.

The exploitation of social media for emergencies requires efforts far beyond the adaptation, customization and effective blending of existing social media processing algorithms in the emergencies and security domains. This is because of the sensitive nature of security incidents and emergencies, their real-time nature, the fact that they are (more) susceptible to social networks compromise and manipulation, the need to combine multiple social media platforms and processing algorithms, and the dynamics of security and emergency management applications.

So far, the following key topics for research were identified by the project, providing a useful overview of the tools/methods lacking in crowdsourcing for the crisis management domain:

- Using behavioral theories in order to understand and model citizens' social mediabehavior before, during and after emergencies.

- Automatic identification and verification of emergency-relevant information from social media in real-time.

- Fast extensible search across multiple social media streams.

- Community analysis across social media sources to infer the sentiments, bias and motivations of reporters and the public during emergencies.

- Behavioral analysis and sentiment identification.

- Topic Based Community Tracking.

- Leveraging Virtual Spaces for evaluating public opinion.

- Plug n' play integration and fusion of social sensors.

Resulting methods will be integrated into the SUPER concept as illustrated in Fig. 1, which provides an overview of how information stemming from social media will be processed and exploited. This can either happen through social sensors (i.e. algorithmic components that process social media and social networking feeds) or based on games, debates, role-playing and other applications that can solicit citizens' feedback in virtual spaces.

Apart from individual social sensors, SUPER will also offer possibilities for combining and reasoning over the outcomes of multiple processing algorithms, with a view to achieving credible results associated with the analysis of citizens' behavior, but also to become able to detect events in nearly real-time. The outcomes of the analysis of social media (based on both social sensors and virtual spaces applications) will be used to drive a number of security and emergency management applications, which will include COP generation, management of real-time operations, policy simulations and ahead planning of strategies associated with security and emergencies incidents.

SUPER will be validated in the scope of different and complementary scenarios, one dealing with emergency management during disasters (i.e. earthquakes) and another dealing with police services. 


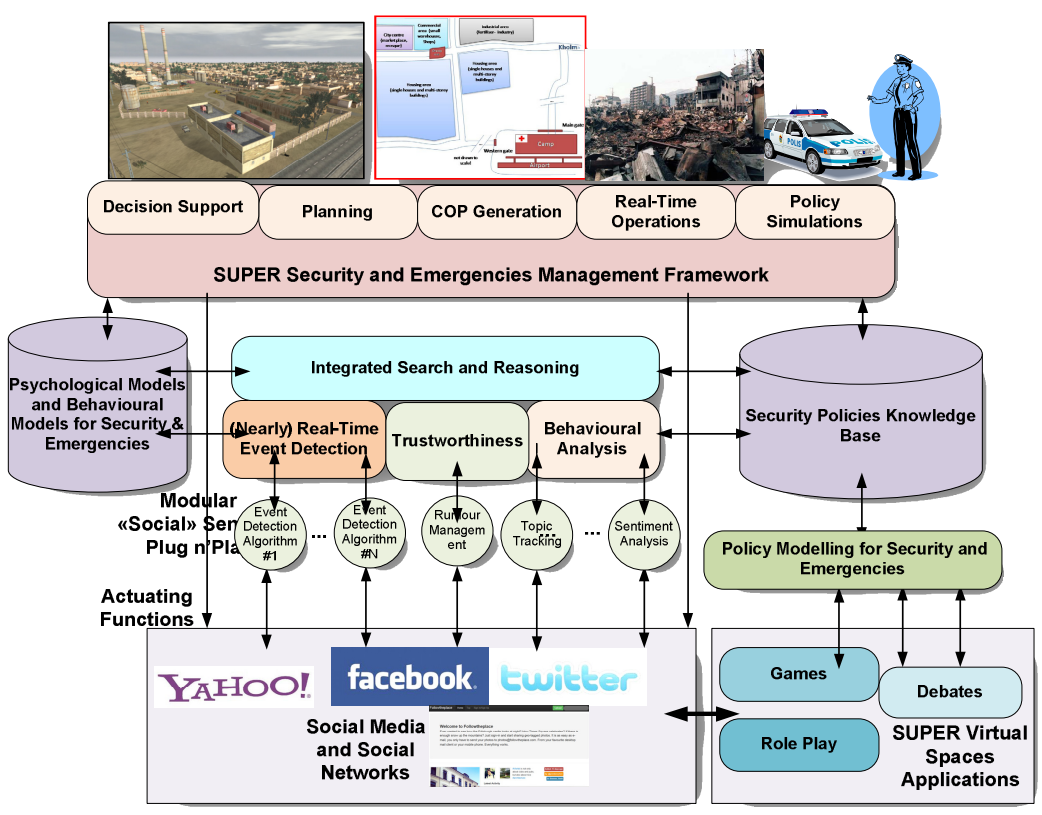

Fig. 1. SUPER Framework overview ${ }^{5}$

\subsection{Environmental Crowdsourcing Techniques for Improved Water Quality in Rural India}

A three-continent research consortium ${ }^{6}$ is evaluating an environmental crowdsourcing technique that relies on inexpensive tests kits (53-cents) that turn purple in the presence of bacterial contamination.

With more than 10 million service points, India's rural drinking water system provides a real monitoring challenge for public health officials. Studied in eight villages during this summer (2014) and scheduled for more widespread evaluation in 2015, the technique could empower residents to check their own water quality and address the problems they find more efficiently. It would be impossible to pay somebody to go out and take all these samples, bring them back to a central lab and process them under controlled conditions.

The set-up is as following: volunteers are given the test kits, which include a test tube containing a material that changes color in the presence of E. coli, a bacterium

5 http://backend.mymeedia.com/data/super-fp7/ c5627e14-ddf0-4b7b-935d-72d30164e5b4.pdf

6 The research has been sponsored by the U.S. Institute of International Education. Partner agencies include the National Environmental Engineering Research Institute (NEERI) in India, and the London School of Hygiene and Tropical Medicine in the United Kingdom. Contacts: John Toon (jtoon@gatech.edu) Research News, Georgia Institute of Technology, 177 North Avenue, Atlanta, Georgia 30332-0181, USA. 
often present in contaminated water. In their homes, the volunteers fill the tubes with water, either from a tap or from a home water storage container. They allow the water to incubate in the test tube overnight, and then use a mobile phone to text a series of numbers indicating, whether the test material remained yellow -corresponding to safe water - or turned purple indicating contamination. A programmed smartphone receives and analyzes the texts, automatically calculating overall water quality by village. The program accounts for errors in reporting by looking for trends in the overall results. We think this may be a scalable model for large-scale environmental monitoring that could be applied in other countries as well.

Beyond improving water quality, the testing program will empower rural citizens of India to take responsibility for their drinking water.

\subsection{Using Crowdsourcing to Fight Climate Change Effects on Agriculture}

Climate change is an important issue today affecting society in various ways. One domain that has a particular interest in understanding long-term changes of weather patterns is the agriculture sector.

Each year, farmers have to purchase and plant seeds that will survive appropriate weather conditions and eventually yield a healthy harvest. An unexpected cold spell or heavy rains can seriously damage crops, financially ruining a farmer or even leading to famines.

In order to combat this problem in developing nations, some scientists start making use of crowdsourcing techniques. As part of the Seeds for Needs initiative, for example, the CGIAR Research Program on Climate Change, Agriculture and Food Security $^{7}$ (CCAFS) has been looking at how to turn farmers into citizen observers.

According to [7] CCAFS's Jacob van Etten, the Seeds for Needs initiative can be understood as a series of projects addressing the issue to give farmers more access to crop varieties and landraces to help them adapt to climate change. As part of that initiative, the CCAFS is currently running a program in Vaishali ${ }^{8}$, a district in India's northeastern Bihar state, to test the robustness of different wheat varieties in this region.

Variety is key here: each kind of crop has unique traits, and some particular crop types may be more suitable than others to a region. Wheat, for example, is highly sensitive to heat during flowering, writes van Etten, so planting varieties of wheat that flower earlier and later than usual can ensure that at least part of the harvest is safe.

Here is where the crowdsourcing aspect of Seeds for Needs comes in. To find out which crop varieties perform best, CCAFS is turning farmers into citizen scientists by asking them to evaluate the harvest. Van Etten describes the project thus:

"Each farmer grows a combination of three varieties drawn from a broader set of ten. The farmer then ranks them according to different characteristics such as early vigour, yield, and grain quality. The idea is to make things as easy as possible for the farmers, and then we, the researchers, use some nifty statistics methods to combine the rankings and share the results with the farmers. With this information, farmers can

\footnotetext{
http://ccafs.cgiar.org/

8 http://ccafs.cgiar.org/atlas-ccafs-sites\#.VIgyTjGG98E
} 
then identify the best varieties for their conditions and preferences. Farmers become citizen crop scientists, actively contributing to science with their time, effort and expertise. In India, 800 farmers are now testing wheat varieties as citizen scientists."

Van Etten laid out his plan for a project like this in [7]. While [7] focused on another region, the underlying process is the same: distribute seeds to a large number of farmers, have them report the harvest results, analyze the data, and share the information among their communities. Adding gamification elements would increase the motivation to participate, the author writes. Farmers in areas with low internet penetration could report their observations through mobile phones.

The goal is to make the crop improvement process cheaper and faster, van Etten claims. The early results in Vaishali have been positive enough for CCAFS to plan two similar programs in East Africa ${ }^{9}$ and Central America. Information above was posted by Anton $\operatorname{Root}^{10}$.

\subsection{Resilience Enhancement by Crowdsourcing and -Tasking}

Crisis and disaster management relies to a large extent on a community of volunteers with a strong commitment and almost professional experience in handling crisis situations. However, due to the increasing mobility of people, the enduring and formal membership in voluntary crisis management has lost its appeal and organizations such as the Red Cross are looking for new ways of loosely binding volunteers to their organizations.

The Austrian research project "Resilience Enhancement by Advanced Communication for Team Austria" 11 (RE-ACTA, [9]) addresses this challenge by defining and testing new processes and workflows for volunteer coordination and developing a platform that supports these workflows in close collaboration with the Austrian Red Cross as end user. The prototype will allow pre-registered volunteers from the "Team Austria" to use their mobile devices for accepting tasks and communicating their results. At the same time, crisis managers will be able to use the platform as a crowdtasking tool for selecting a specific group of volunteers according to their profile and geo-location.

The concept of crowdtasking will be developed even further in the recently started project "Driving Innovation in Crisis Management for European Resilience" 12 $\left(D_{R} I V E R^{13}\right)$. This huge pan-European project intends to establish a set of experimental facilities for testing the crisis management methods and technologies in several EU member states. Within DRIVER, the concepts of crowdsourcing and crowdtasking will be tested in various scenarios and with different volunteer profiles, with the aim

9 http://www.bioversityinternational.org/ e-library/publications/detail/seeds-for-needs-in-east-africa/

10 http://www. crowdsourcing.org/profile/anton-root/5824

11 http://www.kiras.at/gefoerderte-projekte/detail/projekt/ re-acta/; or http://www.reacta.at

12 http: //driver-project.eu/

13 http://driver-project.eu/sites/default/files/driver/files/ content-files/articles/DRIVER\%20Newsletter_1-2014.pdf 
to establish the best practices for increasing the societal resilience through increased involvement of the citizens in the crisis management process. On a technical level, the crowdtasking platform is based on Ubicity platform for data-driven applications. The Ubicity platform is a result of complete rewrite of the experimental Mobile Data Acquisition Platform (MDAF, [8]) backend with the intention to turn the research prototype in a robust product which can be used in crisis management. Ubicity is capable of storing and analyzing large amounts of unstructured and semi-structured geographically and temporally referenced data from multiple domains in near real-time ${ }^{14}$.

In order to ensure privacy of volunteer profiles, the storage of observations is separated from the volunteer data. For the production of situation reports and the monitoring, e.g. of social media, this platform has a generic interface that can be easily connected to different analysis or visualization tools. In order to support a continuous process of communication between crisis managers and volunteers, a web portal provides up-to-date information and allows volunteers to register at any time, as well as to download the mobile application. With the mobile application, volunteers can use their own smartphones to accept or reject tasks that the crisis manager sent out. Fig. 2 illustrates the functional building blocks of the whole platform as they are implemented in the RE-ACTA approach for crowdtasking management [9].

The advantage of such a system is the increased awareness of the situation development in real-time based on spontaneous input from a large number of persons.

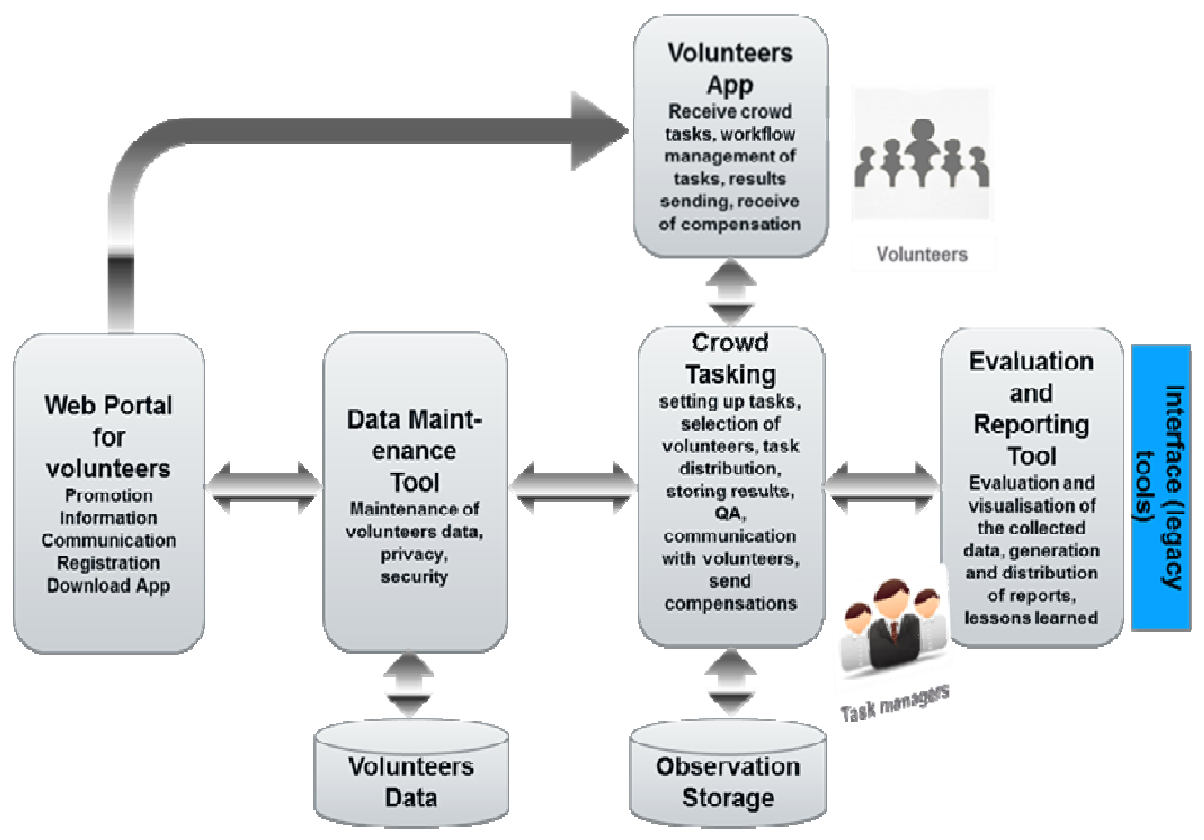

Fig. 2. Building Blocks of the RE-ACTA platform

14 Ubicity details available on github'; https://github.com/ubicity-principal) 
Additionally, spontaneous volunteers can easily be included into the communication process by e.g. registering on a web portal. Spontaneous volunteers are automatically assigned lower level of trust by the crowdtasking system until their profiles are verified by the Red Cross personnel. As a result, they are assigned to easier and less critical tasks and the observations they provide are trusted less than the observations provided by pre-registered volunteers.

\section{$4 \quad$ Key Challenges of Crowdsourcing and Crowdtasking in Crisis Management}

\subsection{Information Extraction and Uncertainty}

Information gathered from citizens is a-priory less reliable than the one gathered from hardware sensors. In spite of the good experiences with use of this information so far, there is no guarantee that the citizens will not post misleading information in the future. The reason for false observations could be lack of knowledge, mischief or even the wish to have some fun [10]. Social data mining brings additional issue of machine-interpreting the messages written by and for humans. As illustrated in section 3.1, this is a difficult and error-prone task. Under the assumption that the misleading information is posted by a minority of users, the quality of information can be improved using statistical methods.

These issues are far less pronounced in a crowdtasking use case, for following reasons:

- Since we have a good knowledge of the users' profile, it's easier to estimate the uncertainty of the received observations.

- Observations are well-defined and therefore easier to process by a machine.

- In case of doubt, additional users can be easily asked to confirm an observation.

\subsection{Self-organization}

During a crisis event, the available resources should be rapidly deployed to the most appropriate tasks. In reality, the globally optimal resource allocation is impossible because the Command Centre has neither a perfect situational awareness nor the capacity to compute the right decision strategy in real-time. The usual strategy for reaching the near-optimal solution in these circumstances is to rely on a combination of written preparedness plans, (incomplete) situation maps and "gut feeling" of the experienced crisis managers [11]. This is incompatible with the self-organization mechanisms of smart mobs and similar social-networking enabled ad-hoc organizations, where decisions are made without prior plans and hierarchical structured organization [12].

Self-organization is implicitly accepted as a way to go whenever the social data mining is used in the context of crisis management (as mentioned in section 3.1). This laisse-faire attitude drastically changes in the moment crisis managers consider the idea of steering and tasking the crowd. Our experience with the development of the crowdtasking system for the Austrian Red Cross (related to section 3.4) shows that the crisis managers consider the self-organization a liability and strive to implement a 
strict task assignment process which avoids the risk of self-organization. In particular, the ad-hoc volunteers should not be given a possibility to directly discuss the situation with other volunteers, invent own tasks or delegate tasks to other volunteers. This has initially led to a design of the system where tasks can be performed exactly once after accepting the assignment.

Apart from the obvious "wouldn't it be better to let them self-organize using your platform than using a platform you have no access to?" objection, we have recently discovered that some level of self-decision must be allowed by design in order to make the crowdtasking more resilient to mobile network disruptions. Since we can't rely on uninterrupted internet connection during the crisis, the ad-hoc volunteers must be pre-assigned with a basic set of tasks appropriate for the crisis situation already in the early warning phase and trusted to perform some of the pre-defined tasks as they fit if the network is interrupted. The next logical step in development would be to enable decentralized phone to phone networking and update the local situation view with the reports from nearby volunteers. In our opinion, the crisis managers will eventually have to accept and even nourish some level of self-organization in crowdtasking platform(s) under their control. Finding a right balance between "total control" and "full self-organization" is a difficult task and the end result is likely to be a platform which allows relatively high level of self-organization while still allowing the crisis managers to request tasks in a top-down manner as well as to interfere with the counterproductive self-organization efforts.

\subsection{Incentives for Participation}

When considering new crowdsourcing systems, perhaps the most important design question pertains to incentive structures. Since crowdsourcing systems are typically open to anyone, and do not rely on contractual relationships, people are not bound to participate. Instead, they must feel somehow compelled to participate, and so the factors that make a given system compelling might also those that make it succeed or fail. In the case of crisis management, the motivation is likely to be high during the crisis and low otherwise, so the main question we need to answer is: how to incite the participation in preparatory phase?

There are many ways to attract crowdworkers, but researchers such as Malone [13] describes three main motivation factors which can be influenced by crowdsourcing platform owners as "money, love and glory" paradigm.

Money: The dominant incentive mechanism on micro-task markets is money. Different markets have different norms for remuneration rates, but higher pay generally gets more workers to do more work more quickly [14]. In some cases, money can be the only motivator that will work. When the work is tedious and unpleasant (such as transcribing pages and pages of hand-written documents), crowdworkers are unlikely to participate unless fair monetary compensation is guaranteed. Money is not necessarily the best way to ensure quality, however. Researchers studying MTurk (Amazon's Mechanical Turk service) have found that higher rates of pay do not necessarily lead to higher quality work as mentioned in [14] and [15]. 
Love: Of course, money is not always required to touch people's heart. If workers simply love the task itself, they will contribute their time for free. Some people love tasks that challenge them and encourage them to help and support, especially in emergency situation or when a disaster occurs.

Glory: Finally, the possibilities to boost own social status by becoming a "hero of the crowdtasking platform" is both a strong incentive and one that is relatively easily realized. Glory incentive relies on measuring the users' performance, e.g. in terms of "points" for performed tasks and giving the visibility to "best" users, e.g. by adding a "beginner"/"experienced"/"hero" attribute to their usernames, distinctively visualizing the different user categories on a map or listing the "heroes of the day" on a special memorial page.

How can we apply these motivation factors to crisis management? A-priory, the volunteers are most likely to be motivated primarily by the factors which we can't influence, such as "moral obligation" or the wish to make the world a better place.

Relying on money as a driving force for ad-hoc volunteers is counterproductive and could be even dangerous, since most of the work in crisis management is already performed by unpaid volunteers and the available budget is tight. However, the recompensation for charges incurred as a result of tasking (e.g. fuel costs) should be considered, if possible. Monetary compensations could play a more prominent role in preparatory phase, when tasks tend to be less exciting and the societal benefits more difficult to understand.

Helping others is a highly emotional activity. Simple ways to boost users' motivation at the "love" level are: pointing out the societal usefulness of the tasks, thanking the users for performed tasks and by visualizing the added value of the ad-hoc volunteers' work during and after the crisis. In a broader context, the "fun factor" can be seen as part of the love incentive. Presenting the tasking as a game (e.g. scavenger hunt tape of game) is therefore a good way to boost volunteers' motivation.

Finally, appealing to the "glory" factor is a good way to additionally strengthen the users' inner motivation for helping others by boosting the social status of the active volunteers. In addition, the "hero of today" score-tables can be used both to advertise the most helpful volunteers and to boost the love/fun factor by adding a touch of competition to the tasking platform.

Even more "glory" can be given to most useful ad-hoc outside of the crowdsourcing platform, e.g. by mentioning the importance of ad-hoc volunteers at post-crisis press event or inviting the most active volunteers to annual Christmas gala dinner. At this level a care must be taken not to over-compensate the ad-hoc volunteers with respect to the crisis management professionals and the institutional volunteers who do not participate in crowdtasking activities.

\subsection{Ethical Considerations}

Crowdsourcing is still an evolving field and many of the ethical implications it raises have yet to be resolved. Since internet has no borders, crowdsourcing could easily evolve into a digital sweatshop with the majority of the work done by "professional crowdworkers" from low income countries such as India [16]. In addition, crowdworkers are often 
given tedious, stupefying tasks that contribute little towards gaining new, marketable job skills. More effort should therefore be undertaken to help crowdworkers develop new, meaningful skills that can be applied to other work domains, as shown in the example "Environmental crowdsourcing techniques for improved water quality in rural India" above.

Unlike remuneration, which is unlikely to play an important role in crisis management, in crowdtasking applications the issue of liability could seriously impede the ability of crisis managers to work with the crowd. Since organizers and volunteers both play an active role in the system, it is unclear to which level they can be made responsible for the accuracy or validity of the crowdtasked information. This becomes important in case of damage or injuries caused by crowdworkers or when decisions affect the safety of crowd members. In order to minimize this type of risks, organizers should assure that tasks are always accompanied with adequate instructions and warnings. In addition, organizers should try to educate the crowdworkers to comply with relevant safety standards and to certify this knowledge, e.g. through micro-learning.

The next issue concerns privacy and misuse of data stored in user profiles or misuse of user tracking information. As a rule, the user profile data should be protected by design and the sensitive data (e.g. health-related) should never be shared with third parties, nor even be accessible to operators. Tracking of the users' positions is a special case. In our opinion, the tracking functionality should only be used with explicit users' consent, and this consent should only be requested for a well-defined area and time duration. As described in [10], the targeted geofencing could even be implemented without the need to track the users.

On a positive side, the issue of falsified profiles (e.g. minors pretending to be 18+) is unlikely to become a serious problem as long as the tasking organizations stick to the policy of validating the users profile in a face to face (offline) session. Finally, parceling of the work into tiny bits is a good way to hide the motives behind task requests from the crowdworkers. A malicious organizer could thus easily trick crowdworkers to participate in unethical activities, such as identifying and tracking of dissidents in a large crowd of people.

\section{Conclusions}

In this paper, we have presented several crowdsourcing use cases, which are relevant for crisis management. The use cases range from merely using whatever information becomes available on the web to actively managing and tasking the crowd. Examples from large-scale crisis incidents and ongoing research projects show that crowdsourcing techniques have a lot to offer in the field of crisis management. In crises situations any environmental, economic and health sector can be affected. However, in order to obtain real-time, accurate and trustworthy information of ongoing crisis incidents and to establish crowdsourcing as additional tool for crisis manager, significant technological and methodological advances are necessary.

Due to the massive user community of general purpose social networking platforms such as Facebook or Twitter, we see on a short time-scale two important developments with high impact in the CDM domain: (1) improved algorithms that accurately detect 
relevant information out of tons of data to a specific topic/theme (social media monitoring) and (2) methods for understanding emotions in the context of short messages or in posts..

On a long-term perspective, crowdtasking has the highest potential for improving the overall societal resilience. However, this is also the form of crowd use which is most difficult to implement and most sensitive to the issues mentioned in the previous section and most difficult to handle at the organizational level.

In principle, designing such a system is relatively simple, but in our opinion crowdsourcing tools are like English: it is easy to develop one, but quite difficult to do it in such a way that it optimally supports crisis managers, while at the same time attracting large numbers of users. In summary, the ideal crowdsourcing application should be designed to support crisis managers in managing issues related to user motivation, to mitigate privacy and ethical issues, as well as to support a right balance between top-down and self-organization of volunteers per design.

Acknowledgments. The research leading to this paper has been performed in the scope of DRIVER and RE-ACTA projects. The DRIVER FP7 project has received funding from the European Union's Seventh Framework Programme for research, technological development and demonstration under grant agreement no 607798. REACTA was funded within the framework of the Austrian Security Research Program KIRAS $^{15}$ by the Federal Ministry for Transport, Innovation and Technology.

Paper authors would also like to acknowledge the work of following developers: Clemens Geyer, Herman Huber and Peter Kutschera who developed the initial version of the MDAF software; Jan van Oort, who started the Ubicity development by rewriting the MDAF backend from scratch; Christoph Ruggenthaler, who is currently the main Ubicity backend developer; and Maria Egly, who is responsible for the mobile application as part of the Ubicity development.

\section{References}

1. Meier, P.: Crisis Mapping in Action: How Open Source Software and Global Volunteer Networks Are Changing the World, One Map at a Time. Journal of Map \& Geography Libraries: Advances in Geospatial Information, Collections \& Archives (May 2012)

2. Bott, M., Gigler, B., Young, G.: The Role of Crowdsourcing for Better Governance in Fragile State Contexts. International Bank for Reconstruction and Development / The World Bank (2014)

3. Sutherlin, G.: A voice in the crowd: Broader implications for crowdsourcing translation during crisis. Journal of Information Science 39(3), 397-409 (2013),

http://jis.sagepub.com/content/39/3/397

4. Morris, R.R., McDuff, D.: Crowdsourcing Techniques for Affective Computing. The Oxford Handbook of Affective Computing (Forthcoming) Edited by R. Calvo, S.K. D'Mello, J. Gratch, A. Kappas, 10.1093/oxfordhb/9780199942237.013.003 (Online Publication Date: April 2014)

5. Howe, J.: The rise of crowdsourcing. Wired Magazine 14(6), 1-4 (2006)

15 http: //www.kiras.at/ 
6. Rheingold, H.: Smart Mobs: The Next Social Revolution. Basic Books (2007), ISBN-13: 978-0738208619

7. van Etten, J.: Crowdsourcing Crop Improvement in Sub-Saharan Africa: A Proposal for a Scalable and Inclusive Approach to Food Security. IDS Bulletin 42, 102-110 (2011), doi:10.1111/j.1759-5436.2011.00240.x

8. Havlik, D., Kutschera, P., Geyer, C., Egly, M.: Geospatial Service Interfaces and Encodings for Mobile Applications in EnviroInfo 2012 Proceedings Part 1: Core Application Areas, Shaker Verlag Aachen, ISBN: 978-3-8440-1248-4

9. Sebald, C., Neubauer, G., Foitik, G., Flachberger, C., Lankmayr, G., Tellioglu, H., Havlik, D.: The RE-ACTA Approach - Resilience Enhancement by Crowdsourcing and Crowdtasking. Poster at the 11th International Conference on Information Systems for Crisis Response and Management, ISCRAM 2014, May 18-21. Penn State University, University Park (2014)

10. Havlik, D., Egly, M., Huber, H., Kutschera, P., Falgenhauer, M., Cizek, M.: Robust and trusted crowd-sourcing and crowd-tasking in the future internet. In: Hřebíček, J., Schimak, G., Kubásek, M., Rizzoli, A.E. (eds.) ISESS 2013. IFIP AICT, vol. 413, pp. 164-176. Springer, Heidelberg (2013)

11. Deri, O., Havlik, D., Rafalowski, C.: Accidental spillage from a container at large city port (Israel). In: Havlik, D., Dihé, P., Frings, S., Steinnocher, K., Aubrecht, C. (eds.) CRISMA Catalogue. CRISMA consortium (2014),

https://crisma-cat.ait.ac.at/print/188 (retrieved on December 11, 2014)

12. Havlik, D., Javier, S., Granell Carlos, G., Middleton, S.E., van der Schaaf, H., Berre Arne, J., Pielorz, J.: Future Internet enablers for VGI applications in EnviroInfo 2013 Proceedings Environmental Informatics and Renewable Energies. In: 27th International Conference on Informatics for Environmental Protection - Informatics for Environmental Protection, Sustainable Development and Risk Management - Part I and II, pp. 620-630 (2013) ISBN 978-3-8440-1676-5

13. Malone, T.: Harnessing Crowds: Mapping the Genome of Collective Intelligence. MIT Sloan Research (2009)

14. Mason, W., Watts, D.J.: Financial incentives and the "performance of crowds". In: Proceedings of the ACM SIGKDD Workshop on Human Computation - HCOMP 1909, p. 77 (1909), Presented at the ACM SIGKDD Workshop, Paris, France (2009)

15. Rogstadius, J., Kostakos, V., Kittur, A., Smus, B., Laredo, J., Vukovic, M.: An Assessment of Intrinsic and Extrinsic Motivation in Crowdsourcing Markets. In: ICWSM 2011, Presented at the Association for the Advancement of Artificial Intelligence (AAAI), Barcelona, Spain (2011)

16. Fort, K., Adda, G., Cohen, K.B.: Amazon Mechanical Turk: Gold Mine or Coal Mine? Computational Linguistics 37(2), 413-420 (2011) 